\title{
Esophageal perforation during neoadjuvant chemoradiotherapy for esophageal cancer following transcatheter arterial embolization: a case report
}

\author{
Kuo-Wei Lu ${ }^{1}$, Tsung-Yen Wang ${ }^{2}$, Pin-Ju Chen ${ }^{2}$, Yang-Hong Dai ${ }^{3}$, Chih-Cheng Tsao ${ }^{1}$ \\ ${ }^{1}$ Department of Radiation Oncology, Taitung MacKay Memorial Hospital, Taitung, Taiwan; ${ }^{2}$ Department of Nursing, Taitung Mackay Memorial \\ Hospital, Taitung, Taiwan; ${ }^{3}$ Department of Radiation Oncology, Tri-Service General Hospital, Taipei, Taiwan \\ Correspondence to: Chih-Cheng Tsao. Department of Radiation Oncology, Taitung MacKay Memorial Hospital, No.1, Lane 303, Changsha Street, \\ Taitung City 95054, Taiwan. Email: kobeiori25@yahoo.com.tw.
}

\begin{abstract}
Esophageal perforation (EP) is a rare disease mostly caused by iatrogenic endoscopic procedures. Esophageal cancer accounts for ten percent of them, and EP under such circumstance could be more complicated and often leads to poor outcome. Therefore, early recognition is of vital importance to prolong survival. To our knowledge, this is the first case report to propose the possible role of transcatheter arterial embolization (TAE) in EP. A 58-year-old female with stage III esophageal cancer (cT3N1M0) was presented to our emergency room. Four months before, she had received TAE because of tumor bleeding and then underwent neoadjuvant concurrent chemoradiotherapy (CRT) after recovery. Prior completion of CRT, dizziness, vomiting, chest tightness and chest pain developed. On examination, fever up to 38.8 degrees was found, accompanied with increased infiltration over the bilateral lungs on the chest radiograph. Under the initial impression of pneumonia, she was first managed with antibiotics and fluid hydration. However, drop of blood pressure occurred six hours later and emergent computed tomography of chest revealed pneumomediastinum and pericardial effusion. Upper gastrointestinal endoscopy showed severe mucosal breakage and EP was confirmed. Broad-spectrum antibiotics and fluid resuscitation were subsequently initiated. Pericardial window was also performed due to cardiac tamponade and the bacterial culture from the mediastinal fluid yielded Pseudomonas aeruginosa. Placement of metal stent was advised but refused by her family. With supportive care only, the patient died one month later due to progressive sepsis and multiorgan failure. EP may occur during CRT if there are risk factors such as T4 primary tumor, age more than 60 or extracapsular lymph node that involves the esophagus. In our case, we thought previous TAE is another factor that possibly contribute to the EP via generation of ischemia and alteration of healing microenvironment. In this setting, risk of EP should be carefully evaluated when subsequent CRT is attempted.
\end{abstract}

Keywords: Esophageal perforation (EP); esophageal cancer; transcatheter arterial embolization (TAE); chemoradiotherapy; case repot

Received: 19 August 2020; Accepted: 16 March 2021; Published: 30 March 2021.

doi: $10.21037 /$ tro-20-54

View this article at: http://dx.doi.org/10.21037/tro-20-54

\section{Introduction}

Esophageal perforation (EP) is a therapeutic challenge because of its lethality. More than half of the perforations are iatrogenic, and most cases occur during endoscopy (1-6). Other causes include spontaneous rupture
(Boerhaave's syndrome), foreign body ingestion, trauma, and intraoperative injury. Although relatively infrequent, malignancy could cause increased intraluminal pressure, and eventually lead to esophageal rupture. Besides, EP could also occur during or after radiotherapy for esophageal 


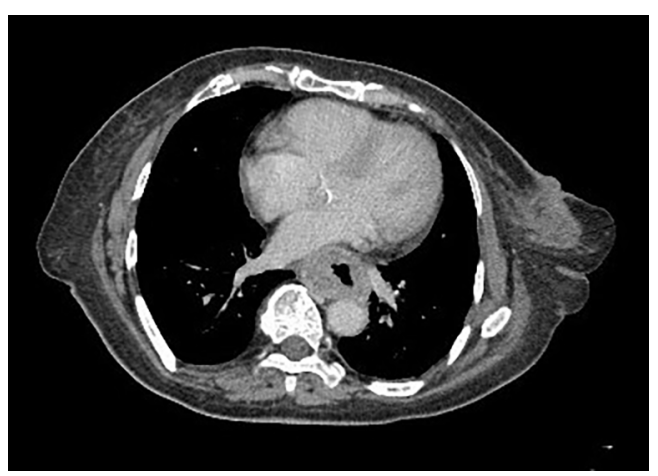

Figure 1 Computed tomography (CT) of chest with contrast at diagnosis.

cancer (7). Chen et al. retrospectively analyzed 322 patients with esophageal carcinoma who received radiotherapy for unresectable, residual or recurrent tumors. They identified that patients with T4 stage, extracapsular lymph node involving the esophagus and re-irradiation were risk factors for esophageal perforation. In the current report, we described a patient with T3 esophageal cancer who incidentally underwent transcatheter arterial embolization (TAE) because of tumor bleeding. This patient had subsequent EP during neoadjuvant chemoradiotherapy (CRT). To our knowledge, TAE used in this clinical setting is rare and might be related to EP in the absence of T4. We present the following case in accordance with the CARE reporting checklist (available at http://dx.doi.org/10.21037/ tro-20-54).

\section{Case presentation}

A 58-year-old female was referred to our hospital. Her present illness could be traced to four months ago when progressive dysphagia and epigastric pain developed, which were accompanied by apparent body weight loss of 20 kilograms in eight months. She visited nearby hospital and a tumor located at the middle third of esophagus was identified through upper gastrointestinal endoscopy. Biopsy was performed and moderately differentiated squamous cell carcinoma was confirmed. Imaging studies with computed tomography (CT) of chest and positron emission tomography confirmed a clinical stage III esophageal cancer (cT3N1M0, according to $8^{\text {th }}$ American Joint Committee on Cancer) (Figure 1). Neoadjuvant CRT followed by surgical intervention was discussed with the patient and was subsequently planned. Unfortunately,

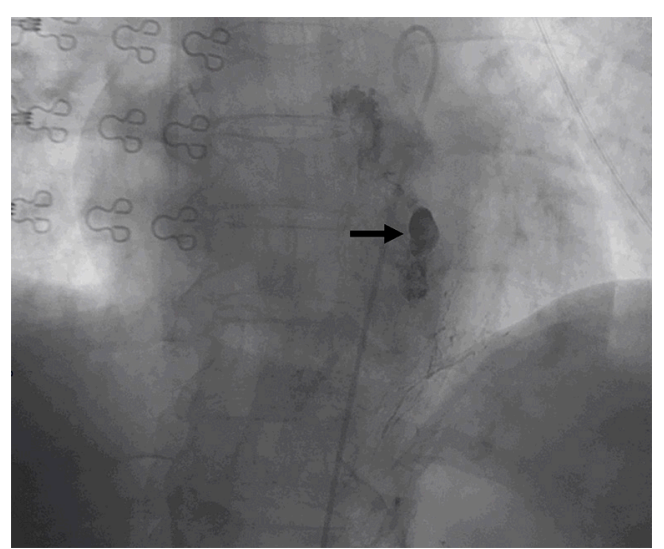

Figure 2 Angiograms showed several hypervascular stains at the middle esophagus with focal extravasation of contrast agent (black arrow).

tumor bleeding was noticed prior to treatment and emergent therapeutic embolization was conducted thereafter at our hospital. The serial angiograms showed several hypervascular stains at the middle esophagus with focal extravasation of contrast agent (Figure 2). The bleeding esophageal artery was originated directly from the aorta. $0.5 \mathrm{~mL}$ of N-butyl-2-cyanoacrylate (NBCA) mixed with $1 \mathrm{~mL}$ of lipiodol was successfully injected. After few days of medical treatment, the bleeding stopped and the hemoglobin level was stabilized. She then underwent CRT with platinum-based regimen (cisplatin and paclitaxel). The radiation dose was planned to 43.2 and 48 gray (Gy) in 24 fractions using intensity-modulated radiotherapy and simultaneous integrated boost technique.

After two courses of chemotherapy and 21 fractions of radiotherapy, she complained of dizziness and weakness for three days, accompanied by nausea, vomiting, poor appetite, chest pain and tightness. Therefore, she was sent to our emergency room. On physical examination, fever up to 38.8 degrees was noticed. Blood pressure, pulse rate and respiratory rate were $100 / 71 \mathrm{mmHg}$, 115/minutes and 20/minutes, respectively. Laboratory results showed hyperglycemia, hyponatremia, anemia and elevated C-reactive protein. Pneumonia was initially impressed and was managed with empiric antibiotics. However, after six hours of conservative management, the systolic blood pressure suddenly dropped to $87 \mathrm{mmHg}$. Isotonic saline infusion and red blood cell transfusion were then performed. Emergent chest CT with contrast disclosed pericardial effusion with focal free air formation within the mediastinum, suggestive of EP (Figure 3). Upper 

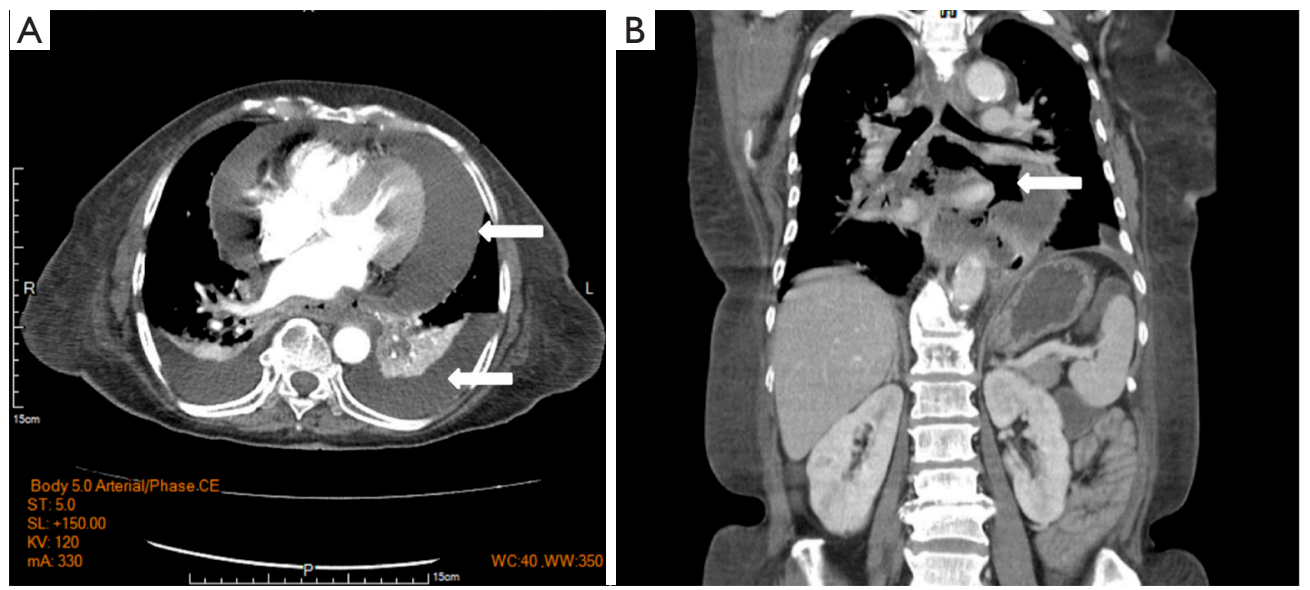

Figure 3 Computed tomography (CT) of chest with contrast at perforation. (A) The axial view of CT disclosed cardiac tamponade and pleural effusion. White arrows indicate these two findings. (B) The coronal view of CT showed pneumomediastinum. White arrow indicates the presence of air in the mediastinum.

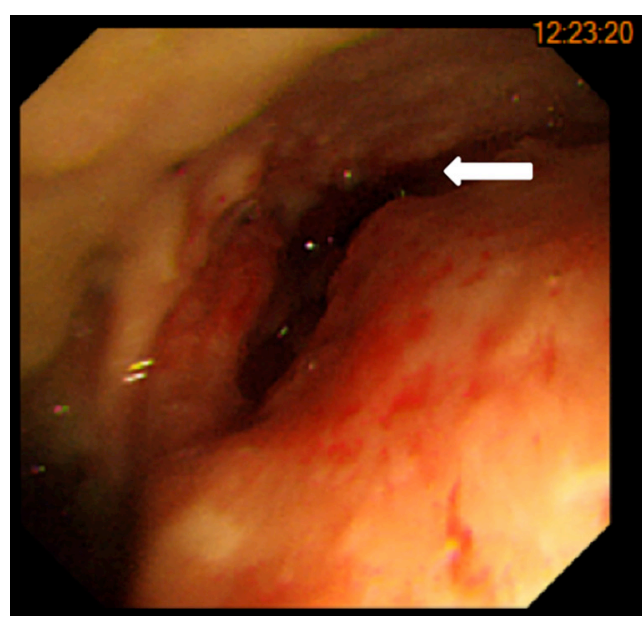

Figure 4 Upper gastrointestinal endoscopy showed ruptured esophagus with adjacent unhealthy mucosa. White arrow indicates the site of rupture. gastrointestinal endoscopy identified a huge ulcerative mass, comparable with the CT findings (Figure 4). The patient was intubated and subsequently managed at our intensive care unit. Pericardial window was performed due to cardiac tamponade. Bacterial culture of the pericardial fluid yield positive Pseudomonas aeruginosa. After discussion with the patient and her family, metal stent for the EP was refused. They selected nonoperative management and supportive care. The patient's condition exacerbated due to progressive sepsis and died one month later due to multiorgan failure. All procedures performed in studies involving human participants were in accordance with the ethical standards of the institutional and/or national research committee(s) and with the Helsinki Declaration (as revised in 2013). Written informed consent was obtained from the patient. Detailed timeline was shown in Figure 5.

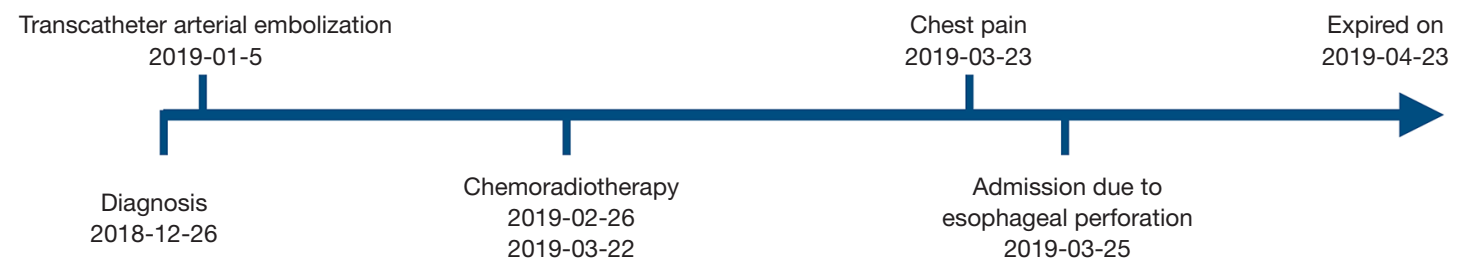

Figure 5 Brief timeline of this case. 


\section{Discussion}

Despite current advances in medicine, EP remains a challenging and life-threatening emergency. The mortality rate was high, with an estimation of $12 \%$ and mean hospital stay of 33 days (8). Due to its rarity and lethality, prospective evaluation of the etiology and natural course is restricted and most data is derived from retrospective series or national studies (9).

The causes of EP are heterogeneous, which comprise a spectrum of conditions ranging from endoscopic instrumentation to spontaneous rupture. Among them, esophageal cancer accounts for ten percent (10). And in esophageal cancer patients, the incidence of EP was about five percent (7) and could rise to $20-33.3 \%$ in the setting of re-irradiation $(7,11)$. Liedman et al. previously reported six cases of middle and lower third esophageal cancers with EP (12). All EPs in their study were caused by barogenic trauma during or after endoscopic dilatation. Beside the increased intraluminal pressure during procedure, spontaneous perforation has also been reported for esophageal cancer $(13,14)$. Chen et al. analyzed the largest number of EP from esophageal cancer, and reported that $5.6 \%$ EP occurred during (10 cases) and after (8 cases) conformal radiotherapy, with (9 cases) and without concurrent chemotherapy (7). After multivariate adjustment, they identified that age less than 60, T4 tumor, extracapsular lymph node involving the esophagus and re-irradiation were associated with a higher risk of EP. They thought younger age could enable patients to undergo CRT, which could accelerate tumor shrinkage while normal tissue repair is suppressed. Additionally, a fistula may form during the tumor shrinkage. In a study by Ohtsu et al., EP occurred in five of 54 patients during CRT and all of them had T4 lesions and fistulas (15). Taken together, it seems the presence of T4 lesions and extracapsular lymph node, which have invaded adjacent tissues, exposes esophageal cancer patients to a higher risk of EP during the cytotoxic treatment.

Concurrent with radiotherapy, the normal tissue repair could be further suppressed, which may lead to hypoplasia of esophageal mucosa in the radiation field $(16,17)$. Additionally, our patient underwent TAE because of episodes of tumor bleeding, which may alter the microenvironment and aggravate the hypoplasia. Among the 18 cases of EP reported by Chen et al., 7 patients experienced EP at the first time of CRT; 4 patients were T4 and 2 patients were T3. For those with T3, extracapsular lymph nodes invading the esophagus was both noted. When examining the patient in our case report, she didn't have T4 disease or ENE involving the esophagus. Considering the incidence rate of $5.6 \%$ and no other risk factors, TAE is the most possible culprit in inducing EP in this patient. In fact, TAE with NBCA has been used for esophageal bleeding for many years $(18,19)$. The use of NBCA is advantageous for patients with coagulopathy and it has high success rate of occlusion. Despite being mentioned as a possible concern, there has been no formal report about ischemic injury or subsequent perforation related to NBCA (18). Nevertheless, in the management of malignancy, healthy tissues are almost always affected and the extent of adverse effects depends on the complex interaction of separate therapeutic modalities. Therefore, we thought the patient's EP might be caused by the interplay of tumor shrinkage during CRT and the ischemia induced by embolization of esophageal artery, which precipitated the spontaneous EP. However, this requires further prospective study for confirmation.

Early diagnosis and timely management of EP are challenging, but both of them are crucial for managing patients (20-22). Diagnosis can be difficult, due mainly to variable and non-specific symptoms, and should consist of repeated and detailed examination when there is persistent clinical suspicion. The pain relevant to EP is often acute and sudden in onset, with radiation to the back or to the left shoulder, which is followed by vomiting and shortness of breath in about $25 \%$ of patients (23). Diagnosis depends on radiographic evidence, including chest radiograph with water-soluble contrast agent, CT with oral contrast and upper endoscopy (24). There are many treatment options, and a multidisciplinary approach is needed. Medications such as antibiotics, proton-pump inhibitors, and so forth can be administered as necessary. Percutaneous drainage or endoscopic therapeutic procedures should be considered according to the clinical situation.

To our knowledge, this is the first report of spontaneous EP preceded by TAE. Unfortunately, the patient died eventually with nonoperative treatment. In general, EP is a surgical emergency and should be immediately managed regardless of etiology $(25,26)$. Without rapid intervention, leakage of esophageal contents into the mediastinum occurs, causing inflammation, sepsis, multiorgan failure and death $(27,28)$. The mortality rate is nearly doubled from 14 to 27 percent for EP diagnosed 24 hours later, underscoring the significance of early detection (27). Moreover, in the retrospective study from England, 2,564 patients were studied and the author showed that patients with EP related to esophageal cancer were more likely to receive 
supportive treatment and the presence of esophageal cancer was significantly associated with increased 30- and 90-day mortality rates (9). Our patient was confirmed to have EP almost 3 days later and was found to have sepsis due to mediastinal infection, which portended her poor prognosis.

To restore luminal integrity and prevent further extraluminal soilage in patients with advanced mediastinal sepsis or large esophageal defects, endoscopic stent could be used in carefully selected patients (29). For our patient, apparent pneumomediastinum and sepsis necessitated further intervention in addition to the broad-spectrum antibiotics and fluid resuscitation. Despite the estimated poor outcome, maintenance of the esophageal integrity could prevent exacerbation of sepsis and increase the likelihood of recovery and survival. In the setting of esophageal malignancy, though not performed in our case, endoscopic stent should be placed.

Our case might have several limitations. Firstly, early diagnosis was not achieved as she suffered from acute onset of symptoms for three days. Secondly, appropriate management was not performed, even though broadspectrum antibiotics and fluid resuscitation were given. Thirdly, tumor bleeding was underestimated and it could represent a sign of advanced cancer, esophageal stent to avoid any presence of small perforation might be an alternative approach before CRT.

In conclusion, we reported a case of EP during CRT in an esophageal cancer patient. Early diagnosis and timely intervention are important to prolong survival. Here we stressed out its possible relation with TAE and the risk of EP should be carefully evaluated when CRT is attempted.

\section{Acknowledgments}

Funding: None.

\section{Footnote}

Reporting checklist: The authors have completed the CARE reporting checklist. Available at http://dx.doi.org/10.21037/ tro-20-54

Conflicts of Interest: All authors have completed the ICMJE uniform disclosure form (available at http://dx.doi. org/10.21037/tro-20-54). The authors have no conflicts of interest to declare.

Ethical Statement: The authors are accountable for all aspects of the work in ensuring that questions related to the accuracy or integrity of any part of the work are appropriately investigated and resolved. All procedures performed in studies involving human participants were in accordance with the ethical standards of the institutional and/or national research committee(s) and with the Helsinki Declaration (as revised in 2013). Written informed consent was obtained from the patient.

Open Access Statement: This is an Open Access article distributed in accordance with the Creative Commons Attribution-NonCommercial-NoDerivs 4.0 International License (CC BY-NC-ND 4.0), which permits the noncommercial replication and distribution of the article with the strict proviso that no changes or edits are made and the original work is properly cited (including links to both the formal publication through the relevant DOI and the license). See: https://creativecommons.org/licenses/by-nc-nd/4.0/.

\section{References}

1. Bufkin BL, Miller JI Jr, Mansour KA. Esophageal perforation: emphasis on management. Ann Thorac Surg 1996;61:1447-51; discussion 1451-2.

2. Kavic SM, Basson MD. Complications of endoscopy. Am J Surg 2001;181:319-32.

3. Brinster CJ, Singhal S, Lee L, et al. Evolving options in the management of esophageal perforation. Ann Thorac Surg 2004;77:1475-83.

4. Merchea A, Cullinane DC, Sawyer MD, et al. Esophagogastroduodenoscopy-associated gastrointestinal perforations: a single-center experience. Surgery 2010;148:876-80; discussion 881-2.

5. Boeckxstaens GE, Annese V, des Varannes SB, et al. Pneumatic dilation versus laparoscopic Heller's myotomy for idiopathic achalasia. N Engl J Med 2011;364:1807-16.

6. Helton WB, Unnikrishnan R, Gal T. Cervical esophageal perforation and cricopharyngeal dysfunction. Ear Nose Throat J 2011;90:E8-E10.

7. Chen HY, Ma XM, Ye M, et al. Esophageal perforation during or after conformal radiotherapy for esophageal carcinoma. J Radiat Res 2014;55:940-7.

8. Biancari F, D'Andrea V, Paone R, et al. Current treatment and outcome of esophageal perforations in adults: systematic review and meta-analysis of 75 studies. World J Surg 2013;37:1051-9.

9. Markar SR, Mackenzie H, Wiggins T, et al. Management and Outcomes of Esophageal Perforation: A National 
Study of 2,564 Patients in England. Am J Gastroenterol 2015;110:1559-66.

10. Rezapour M, Gerson LB. A Tear in The Tube: Any Improvement for Patients With Esophageal Perforation Over Time? Gastroenterology 2016;150:1245-6.

11. Zhou ZG, Zhen CJ, Bai WW, et al. Salvage radiotherapy in patients with local recurrent esophageal cancer after radical radiochemotherapy. Radiat Oncol 2015;10:54.

12. Liedman B, Johnsson E, Lundell L. Treatment of iatrogenic perforations with covered stents in patients with oesophageal cancer. Eur J Surg 2001;167:672-4.

13. Chou YP, Chanchien CS, Chuah SK, et al. Risk factors for mortality of esophageal perforation: a clinical experience in 32 cases. Journal of Internal Medicine of Taiwan 2002;13:256-62.

14. Griffiths EA, Yap N, Poulter J, et al. Thirty-four cases of esophageal perforation: the experience of a district general hospital in the UK. Dis Esophagus 2009;22:616-25.

15. Ohtsu A, Boku N, Muro K, et al. Definitive chemoradiotherapy for T4 and/or M1 lymph node squamous cell carcinoma of the esophagus. J Clin Oncol 1999;17:2915-21.

16. Stone HB, Coleman CN, Anscher MS, et al. Effects of radiation on normal tissue: consequences and mechanisms. Lancet Oncol 2003;4:529-36.

17. Dormand EL, Banwell PE, Goodacre TE. Radiotherapy and wound healing. Int Wound J 2005;2:112-27.

18. Park JH, Kim HC, Chung JW, et al. Transcatheter arterial embolization of arterial esophageal bleeding with the use of N-butyl cyanoacrylate. Korean J Radiol 2009;10:361-5.

19. Aoki M, Tokue H, Koyama Y, et al. Transcatheter arterial embolization with N-butyl cyanoacrylate for arterial esophageal bleeding in esophageal cancer patients. World J Surg Oncol 2016;14:54.

20. Hermansson M, Johansson J, Gudbjartsson T, et al.

doi: $10.21037 /$ tro-20-54

Cite this article as: Lu KW, Wang TY, Chen PJ, Dai YH, Tsao CC. Esophageal perforation during neoadjuvant chemoradiotherapy for esophageal cancer following transcatheter arterial embolization: a case report. Ther Radiol Oncol 2021;5:4.
Esophageal perforation in South of Sweden: results of surgical treatment in 125 consecutive patients. BMC Surg 2010;10:31.

21. Bhatia P, Fortin D, Inculet RI, et al. Current concepts in the management of esophageal perforations: a twentyseven year Canadian experience. Ann Thorac Surg 2011;92:209-15.

22. Ryom P, Ravn JB, Penninga L, et al. Aetiology, treatment and mortality after oesophageal perforation in Denmark. Dan Med Bull 2011;58:A4267.

23. Mackler SA. Spontaneous rupture of the esophagus; an experimental and clinical study. Surg Gynecol Obstet 1952;95:345-56.

24. Soreide JA, Viste A. Esophageal perforation: diagnostic work-up and clinical decision-making in the first 24 hours. Scand J Trauma Resusc Emerg Med 2011;19:66.

25. Curci JJ, Horman MJ. Boerhaave's syndrome: The importance of early diagnosis and treatment. Ann Surg 1976;183:401-8.

26. de Schipper JP, Pull ter Gunne AF, Oostvogel HJ, et al. Spontaneous rupture of the oesophagus: Boerhaave's syndrome in 2008. Literature review and treatment algorithm. Dig Surg 2009;26:1-6.

27. Shaker H, Elsayed H, Whittle I, et al. The influence of the 'golden 24-h rule' on the prognosis of oesophageal perforation in the modern era. Eur J Cardiothorac Surg 2010;38:216-22.

28. Vallbohmer D, Holscher AH, Holscher M, et al. Options in the management of esophageal perforation: analysis over a 12-year period. Dis Esophagus 2010;23:185-90.

29. Fischer A, Thomusch O, Benz S, et al. Nonoperative treatment of 15 benign esophageal perforations with self-expandable covered metal stents. Ann Thorac Surg 2006;81:467-72. 